\title{
Visual display in the multipurpose laboratory: The benefits of video scan-conversion
}

\author{
JAMES W. LIVINGSTON, JR. \\ Rutgers University, New Brunswick, New Jersey 08909
}

\begin{abstract}
A visual display system, based upon the PEP-400 video scan-converter, is described. For a cost of about $\$ 5,600$, including nine video monitors, a laboratory can provide flexible, computer-generated, alphameric, and graphic displays simultaneously to eight subjects. Some performance results are given, as is discussion of interfacing and software required for a DEC lab system.
\end{abstract}

I would like to describe a computer-controlled system for presenting visual stimuli simultaneously to multiple subjects. The system design is predicated on the assumption that the equivalent of a dynamic, near tachistoscopic slide projector is adequate for the purposes of the laboratory; this is our situation at the human experimental laboratory of Livingston College. In the description to follow, I will try to show that the device which constitutes the core of our system has quite general utility for information display, especially in multi-user, teaching, and research laboratories. First, I will note the most obvious alternative which might be considered in such a situation, then move on to the specifics of our device and its software, and finally compare briefly the cost of that system with that of the most viable alternative.

In order properly to establish the context for this description, it is helpful to relate the objectives that my colleagues and I sought to satisfy in proposing the laboratory system. We intended to support demonstration experiments for an undergraduate, large-enrollment course in human learning, perception, and other cognitive processes. Faculty and student research was to be supported if the available resources were adequate to the needs of the experimenter, or if he/she chose to augment the existing system. Our main requirement for a visual display system was that it be capable of delivering both textual and graphic output to multiple subject stations, with each possibly isolated from the others. Further, it had to be the least expensive system that could accomplish those goals with acceptable precision of control.

The system we first conceived included a direct-view storage tube (DVST), the Tektronix 613, driven by the display controller (LPSVC) of our laboratory interface subsystem (DEC LPS-11). The DVST was to be scanned by a standard CCTV video camera, the output of which would be distributed with standard CCTV hardware. No serious thought was given to using multiple CRT or DVST displays, since any appreciable number of those

Construction of the Human Experimental Psychology Laboratory at Livingston College of Rutgers University was supported in part by NSF 1SEP Grant GY-10170. would have been prohibiiiveiy expensive, if not necessarily more costly to interface to the computer system. With contemporary (1975) LSI technology, other options could be considered, e.g., large buffer memories and direct video display circuitry; the best display system we could locate in the summer of 1972, however, was the Princeton Electronics Products PEP-400 graphics terminal (PEP-400). As will subsequently become clear, I believe it still has an edge on competing technologies.

The heart of the PEP 400 is the Lithicon (registered trademark), a silicon-target storage tube which is written upon, scanned (read), and erased by a single electron gun. Conceptually, it is best regarded as a gray-scale storage tube and a video camera in a vacuum-sealed package. The PEP-400 is commonly found, among other places, buried in the baggage scanning equipment one encounters in large airports and in medical ultrasound imaging systems. The properties of the PEP-400 which make it most useful to us are not only those which make it look like a camera and DVST; it is also so packaged as to permit external control of its various states, which we provide with the LPSVC. The LPSVC has considerable flexibility built into it, since it is intended to drive a variety of widely used display devices. Included in its control register are bits for controlling mode (store, nonstore), channel $(1,2)$, color (red, green) and the like. The external connector for the LPSVC carries standard (TTL) logic signals corresponding to the register bits; the external connector on the PEP 400 has the same kind of signals, so the two are relatively easy to interface. In addition to control lines, the PEP's external connector has signals which indicate the states of both the target (read, write, or erase) and the internal video synchronization generator (in vertical retrace or not). These may be returned to the LPSVC for proper synchronization of the computer and display. In view of the correspondence between our requirements and the characteristics of the PEP, we elected to use it and eight monitors as the laboratory display system.

As is well known, deciding on the hardware configuration is less than half the necessary decision making. The specific means by which the device was to be controlled had still to be chosen. The choice of a software system is 
best understood by recalling our original conception of the laboratory and its intended user community.

We decided it was important to utilize a widely known, high-level language for applications, since the programming manpower needed to construct a language for our specific situation was unavailable, and the laboratory was intended to be, simultaneously, a teaching tool for graduate instruction, a faculty research resource, and a demonstration facility for undergraduate courses. There are only two languages which satisfy the requirements of wide acceptance and procedure orientedness, and are supported by affordable laboratory-scale computer systems: BASIC and FORTRAN IV. The former has veen adyocated as the language of choice for multi-user laboratory systems by some, most recently Castellan (1975). While it is true that BASIC is easier to learn and supported by smaller computer systems than is FORTRAN IV, it is also slower in execution and somewhat less readily extended for laboratory control and data gathering than the latter (see Donchin \& Hefly, 1975 , for additional discussion). In view of our hardware configuration's capacity, we elected to employ FORTRAN IV for applications programming, and to extend it by producing a system of FORTRAN-callable subroutines for control and data recording. Indeed, we had to write subroutines for all the functions of the LPS-11; our unit was delivered well before DEC had produced their own software for that subsystem.

A substantial subset of our extensions is used for controlling the PEP-400. The subroutine calls are summarized in Table 1.

The table indicates that requirements for the PEP-400 fall naturally into three categories: display control, graphic write and erase, and alphameric write and erase, with "carriage control." The set of subroutines we have developed represents a compromise between economy of core and power, or ease of use. As a software system, it is reported to be relatively simple to use for both novice and advanced FORTRAN IV programmers.

In our applications, the PEP-400 is used to distribute both graphic and textual material to individual stations. The control software, together with some of the adjustment requirements that have proved necessary for carrying out the operations desired, placed limits on our (ancient) device that should not be expected with current models. As presently configured, the system performs as follows: The capacity of the display, in software-generated 5 by 7 dot-matrix characters, is 25 lines of 36 characters each, per screen. Character writing is table-driven, so changing the character set is a matter of constructing a new table. As a point-plot display, the PEP 400 is defined by the LPSVC's 12-bit DACs to be a 4096 by 4096 array. In our present implementation, we can display no more than about 410 distinct lines of 410 distinct points each. In writing speed, we do somewhat better. The PEP's character rate, with our software, is about 1,200 characters/sec. Assuming an average of 20 points/character, it yields a point-plotting rate of about $24 \mathrm{~K}$ points/sec, which is about $36 \%$ of the device's specified capacity. The monitors, moderately goodquality Panasonics (TN-95) with 22.86-cm (diagonal measure) screens, provide quite readable displays at the present character density. The one really troublesome limit that the early model imposes is its full-screen erase interval, about $1.5 \mathrm{sec}$. The current model of the same device, the PEP $400 R$, eliminates the probiem nicely, but we cannot afford to purchase one. It does a fullscreen clear in $66 \mathrm{msec}$. As it is, a main program can operate in parallel with screen clear, but the minimum interval puts a lower bound on our intertrial interval that sometimes causes problems.

To provide criteria for evaluating the cost of a system that can deliver both alphameric and graphic information to eight display screens, note first that good random-access CRT displays are priced from $\$ 700$ each and must be refreshed continuously. DVSTs are at least $\$ 2,500$ for as near an equivalent display area. A single DVST and video camera with ample fidelity can be purchased for about $\$ 3,500$, but to that cost must be added the price of a light-tight enclosure for the pair of

Table 1

FORTRAN Subroutine Calls for PEP-400 Operation

\begin{tabular}{|c|c|c|}
\hline$\overline{\text { GROUP }}$ & CALL & FUNCTION \\
\hline Control & $\begin{array}{l}\text { CALL SCDON } \\
\text { CALL SCDOFF } \\
\text { CALL SCDCLR } \\
\text { CALL SCDVWT }\end{array}$ & $\begin{array}{l}\text { Initiates video output (read). } \\
\text { Terminates video output. } \\
\text { Generates full-screen erase. } \\
\text { Waits on vertical retrace signal from the PEP- } 400 \text { before returning control to caller. }\end{array}$ \\
\hline Graphic & $\begin{array}{l}\text { CALL SCDMOV(IX, IY) } \\
\text { CALL STLINE (IX, IY) } \\
\text { CALL SCWRIT(N, IX, IY) } \\
\text { CALL SCERAS (N, IX, IY) }\end{array}$ & $\begin{array}{l}\text { Sets the software "cursor" to the coordinates IX, IY. } \\
\text { Draws straight line from software cursor to IX, IY. } \\
\text { Plots } N \text { of the points in the arrays IX, IY. } \\
\text { Erases selectively } N \text { of the points in the arrays IX, IY. }\end{array}$ \\
\hline Alphameric & $\begin{array}{l}\text { CALL SCREST } \\
\text { CALL SCDCR } \\
\text { CALL SCDLF } \\
\text { CALL SCBKSP } \\
\text { CALL SCALPH(N,STRING) } \\
\text { CALL SCSCUR(IX,IY) }\end{array}$ & $\begin{array}{l}\text { Restores software character "cursor" to upper left corner of the display. } \\
\text { Returns the cursor to the leftmost margin of the current line. } \\
\text { Advances the cursor to the next line. } \\
\text { Backspaces the cursor one character. } \\
\text { Writes the first N of the characters whose ASCII codes are in the array STRING. } \\
\text { Sets the character cursor to coordinates IX, IY. }\end{array}$ \\
\hline
\end{tabular}


devices, as well as a computer-controlled switch for the camera output. The current model of the PEP, the PEP$400 \mathrm{R}$, can be purchased for $\$ 3,750$. But about $\$ 40$ must be added for the LPSVC.PEP interface. I believe that any other vendor's display controller would be no more difficult to interface to the PEP-400 than is DEC's, but I have not specifically researched the question. Those users with greater resolution demands than can be filled by the PEP-400R might consider its big brother, the PEP-402R. Described specifications on the latter claim about 1,500 visible line (diagonal) resolution, to the former's 1,000. Also available are high-resolution scan rates, although monitors that can be driven by those rates become very costly.

\section{SUMMARY}

The PEP supports quite flexible visual stimulus output to eight subject stations, under the control of FORTRAN IV callable subroutines. It does so with relatively precise control of stimulus onset and offset, and it can be duplicated for a cost, including monitors equivalent to ours (at a cost of about $\$ 200$ each), of approximately $\$ 5,600$. I expect that a similar scan converter offered by Hughes Electronics might be readily adapted to the system described here. The Hughes devices probably bracket the PEP-400R in both price and performance. One model, which costs less (about $\$ 2,300$ ) than the PEP-400R performs less well; the model that performs similarly costs about $\$ 4,400$. The software discussed above is written in DEC PDP-1 1 MACRO and is available to anyone who will send a DECPACK (RK05 cartridge) on which to copy it.

\section{REFERENCES}

Castellan, N. J., JR. The modern minicomputer in laboratory operation. American Psychologist, 1975, 30, 205-211.

Donchin. E.. \& Hefly. E. Minicomputers in the signal-averaging laboratory. American Psichologist. 1975. 30, 299-312. 